\title{
Dynamic Analysis of Three Bar Tensegrity Structure under Compressive Load for Robotic Application
}

\author{
Asif Raza ${ }^{\text {a }}$, Ani Luo ${ }^{\text {a }}$, Muhammad Basit Chandio ${ }^{\text {a }}$, Sanaullah ${ }^{\text {a }}$, Zubair Ali Shah ${ }^{\text {b }}{ }^{\mathrm{c}}$ Ghazanfar Mehdi ${ }^{\mathrm{b}}$ \\ ${ }^{a}$ College of Mechanical and Electrical Engineering, Harbin Engineering University, China \\ ${ }^{\mathrm{b}}$ College of Power and Energy Engineering, Harbin Engineering University, China
}

\begin{abstract}
Tensegrity structures which are comprised of bars and strings are very lightweight structures and they have the capability to deform and remain stable. For these properties they have been utilized in various fields of engineering for various purposes. One of the potential application of tensegrities is in the area of robotics. Three bar tensegrity is a basic unit structure, the dynamics of which can be utilized for tensegrity based robots. Due to its similar geometrical shape and easy control of strings, and by the application of inward compressive load, the movement of whole robot can be controlled. In this paper, the dynamics of three bar tensegrity structure has been studied under compressive load. The mathematical model for the structure has been built; and node matrix and connectivity matrix have been defined to describe the structure. The simulation has been performed on ADAMS software to analyze the movements of bar and deformation in strings under the effect of compressive load. All the strings have been grouped according to the similar deformation experienced by them. In addition to that, compression and twisting of top layer of the structure has been investigated. The obtained results provide the base for the construction of extremely lightweight robotic structure
\end{abstract}

Keywords- Three bar tensegrity, Dynamics, Compression, Robotic structure, ADAMS

Date Received 25-10-2020

Date Accepted 02-11-2020

Date Published 18-12-2020

\section{INTRODUCTION}

$\mathrm{T}$ HE word 'tensegrity' is a combination of two words: tension and integrity [1], which implies that a kind of structure that have a certain strength under the action of tension force. Tensegrity is a new type of structure which is composed of strings bearing tensile loads and bars possessing compressive loads thus forming a stable structure. Guest [2] gave the similar definition of tensegrity but also explained that the compression members (bars) should not be in contact with each other.

Tensegrity structures found numerous applications in the field of architecture, medical and engineering; although it was first considered as part of arts only. David [3], [4] using tensegrity concepts designed long-span structure which is now known as Georgia Dome, was practically made in U.S as main hall for 1996 Atlanta Summer Olympic Games. Tensegrities are considered as smart structures and their property of being lightweight and stiff structure, they have been used as deployable structures, experimentally demonstrated by NASA [5], [6] Principles of tensegrity have also been widely interpreted in the field of biomedical engineering. Human skeleton which is composed of 206 bones can be modelled as bars that are being pulled by the tension force of ligaments, tendons and muscles making a stable vertical structure [7]

Zhang and other authors [8] have defined a reliable and simple method for prismatic tensegrity structure configurations, members and nodes of which possessing dihedral symmetry. R. Connelly [9] has explained finite number of configurations for obtaining stable structures but they must maintain their shape when they are in motion in three-space. Skelton [10] proposed that variations in lengths of strings can cause change in shape of the structure, while retaining the tensegrity conditions of equilibrium and stability. Basit [11] analyzed the dynamics of six bar ball type tensegrity structure by applying compression and expansion forces and observed a dynamic similarity between certain groups of bars and strings. Luo and other authors [12] studied the driving of six bar tensegrity robot and using the simulation and by building up a physical model they verified that tensegrity robot motion can be achieved by altering the bar's length but they did not study the change in strings' length to shift center of gravity of robot to cause the motion. Chandana [13] used genetic algorithm in simulation for 3-strut tensegrity robot to achieve forward gait by controlling length of vertical strings of the structure.

Tensegrity structures are highly tunable and they can show variations in shape easily under the effect of external force or torque, therefore they are being employed as deployable structure applications and also they can be used as robotic structure. In our present work, we have done dynamic analysis of the three-bar prismatic tensegrity structure under compressive load. Firstly, the connectivity and geometry of the structure has been defined and mathematical model has been formed. Secondly, simulation model has been built on ADAMS software and motion of bars and deformation in strings have been analyzed along with compression and twisting of top layer of the structure. Finally, the feasibility of 
our dynamic analysis in the field of robotics is discussed.

\section{MAthematical Model of ThreE BAR TenSEGRITY STRUCTURE}

Referring to Fig. 1, the prismatic bar tensegrity structure can be considered as two regular polygons separated by a definite height. Both the polygons are at a rotated angle with respect to each other. The vertices of the polygons form the nodes of the structure which are connected by bars and strings. The three bar prismatic tensegrity structure consists of three bar and nine strings. There are six horizontal strings; three on each bottom and top side of the structure. The remaining three are vertical strings connecting the top and bottom triangles by their vertices, thus forming a stable tensegrity configuration.

For mathematical modelling, the number of bars is denoted by $p$ and the circle formed by vertices of triangle has radius $r$ and the triangles are separated by a height $h_{0}$ as shown in Fig. 1 .

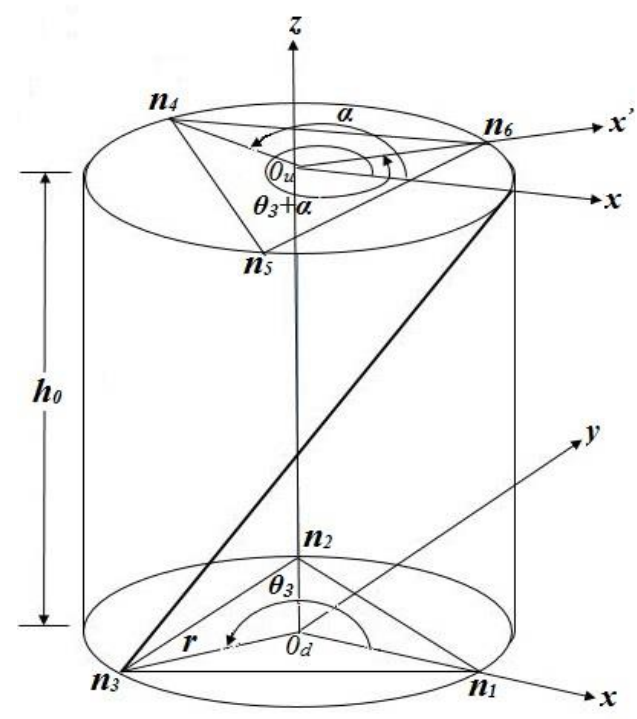

Fig. 1. Mathematical model

Each node of the structure can be denoted by $n_{i}$ therefore the co-ordinates of bottom nodes are given by,

$$
\left[\begin{array}{l}
x \\
y \\
z
\end{array}\right]=\left[\begin{array}{c}
r \cos \theta_{i} \\
r \sin \theta_{i} \\
0
\end{array}\right]
$$

The co-ordinates of top nodes are also obtained similarly but there must be addition of rotation angle $\alpha$

$$
\left[\begin{array}{l}
x \\
y \\
z
\end{array}\right]=\left[\begin{array}{c}
r \cos \left(\theta_{i}+\alpha\right) \\
r \sin \left(\theta_{i}+\alpha\right) \\
h_{0}
\end{array}\right]
$$

Where $\theta$ is the angle between the nodes as shown in Fig.1. The value of angle $\theta \mathrm{i}$ is given by,

$$
\begin{aligned}
& \theta_{i}=\frac{2 \pi}{p}(i-1), \mathrm{i}=1,2,3 \\
& \theta_{i}=\frac{2 \pi}{p}(i-4), \mathrm{i}=4,5,6
\end{aligned}
$$

The angle $\alpha$ is the rotation angle which also defines $p-1$ different species of the structure. According to Skelton [14] and Motro [15] for a stable configuration it is given as follows:

$$
\alpha=\frac{\pi}{2}+\frac{j \pi}{p}, \mathrm{j}=1,2, \ldots, \mathrm{p}-1
$$

The nodes obtained from above formulations can then be arranged column wise in a matrix to form the node matrix as follows:

$$
N=\left[n_{1}, n_{2} \ldots n_{6}\right]_{3 \times 6}
$$

To represent the connection of each member of the tensegrity structure with each node; bar connectivity matrix and string connectivity matrix are formed according to the following rule:

$$
C_{(i, j)}=\left\{\begin{array}{cc}
-1 & \text { if }(\mathrm{i}, \mathrm{j}) \text { is the starting node of the considered member } \\
1 & \text { if }(\mathrm{i}, \mathrm{j}) \text { is the ending node of the considered member } \\
0 & \text { if }(\mathrm{i}, \mathrm{j}) \text { node has no connection considered member }
\end{array}\right.
$$

Combining the Bar connectivity matrix and String connectivity matrix, the overall Connectivity matrix becomes,

$$
C=\left[\begin{array}{l}
C_{B} \\
C_{S}
\end{array}\right]
$$

Lastly, the Bar matrix and String matrix are defined according to the following equations:

$$
\begin{gathered}
B=N C_{B}^{T} \\
S=N C_{S}^{T}
\end{gathered}
$$

For our model, we considered $j=1$ type three bar tensegrity structure and selected radius as $200 \mathrm{~mm}$ and height as 500 $\mathrm{mm}$, we obtained the bottom and top node co-ordinates which are arranged column wise in the node matrix $\mathrm{N}$ as follows:

$$
\begin{aligned}
& \begin{array}{llllll}
\mathrm{n}_{1} & \mathrm{n}_{2} & \mathrm{n}_{3} & \mathrm{n}_{4} & \mathrm{n}_{5} & \mathrm{n}_{6}
\end{array} \\
& \boldsymbol{N}=\left[\begin{array}{cccccc}
200 & -100 & -100 & -173.2 & 0 & 173.2 \\
0 & 173.2 & -173.2 & 100 & -200 & 100 \\
0 & 0 & 0 & 500 & 500 & 500
\end{array}\right]
\end{aligned}
$$

Bar connectivity according to rules mentioned in (7) is given as follows:

$$
\boldsymbol{C}_{\boldsymbol{b}}=\left[\begin{array}{cccccc}
-1 & 0 & 0 & 1 & 0 & 0 \\
0 & -1 & 0 & 0 & 1 & 0 \\
0 & 0 & -1 & 0 & 0 & 1
\end{array}\right]
$$

And string connectivity is given by, 


$$
\boldsymbol{C}_{s}=\left[\begin{array}{cccccc}
-1 & 1 & 0 & 0 & 0 & 0 \\
0 & -1 & 1 & 0 & 0 & 0 \\
1 & 0 & -1 & 0 & 0 & 0 \\
0 & 0 & 0 & -1 & 1 & 0 \\
0 & 0 & 0 & 0 & -1 & 1 \\
0 & 0 & 0 & 1 & 0 & -1 \\
-1 & 0 & 0 & 0 & 0 & 1 \\
0 & -1 & 0 & 1 & 0 & 0 \\
0 & 0 & -1 & 0 & 1 & 0
\end{array}\right]
$$

Bar matrix and String matrix are not shown here because they can be simply obtained by the multiplication of node matrix by the transpose of Bar connectivity matrix and String connectivity matrix respectively. Based on the mathematical model, a MATLAB code generated the three bar tensegrity model as shown in Fig. 2 where the thick blue lines represents bars and the thin red lines represents strings, authenticating mathematical model.

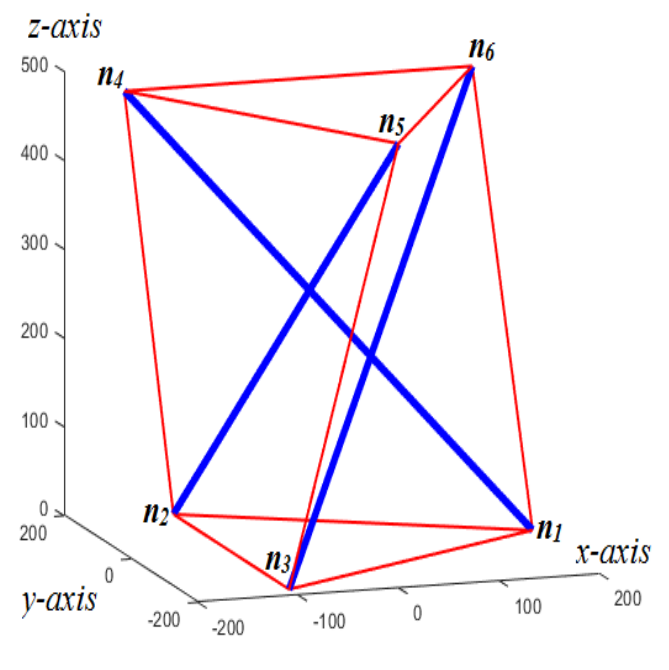

Fig. 2. Three bar tensegrity structure

After the formation of mathematical model, we proceed for the dynamic analysis of three bar tensegrity structure in the next section.

\section{SIMULATION}

Using the node coordinates mentioned in node matrix $\mathrm{N}$ (11) and following the connectivity pattern of members mentioned in Bar connectivity (12) and String connectivity matrices (13) a simulation model was built in ADAMS software for analyzing the effect of compressive forces on the bars and strings. Three forces of $100 \mathrm{~N}$ were applied on each top node that is $\mathrm{n} 4, \mathrm{n} 5$ and $\mathrm{n} 6$. The bottom nodes were fixed to a plate using spherical joint and the plate on which nodes were attached was fixed to the ground thus structure will be compressed upon application of the load. The simulation model is shown in Fig. 3, the blue color represents bar while the strings are represented as springs of red colors. The node numbers are mentioned on each node while the string and bar members are shown by arrow

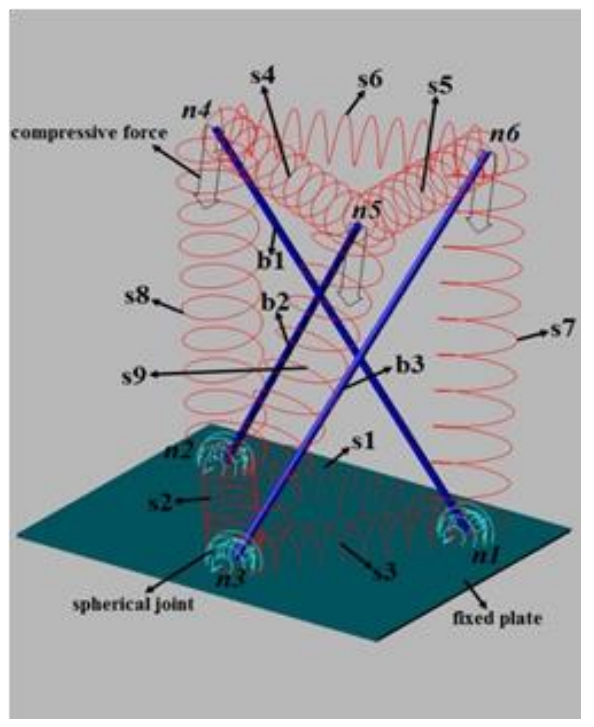

Fig. 3. Three bar tensegrity structure before applying compressive load

The parameters for simulation are shown Table I below.

TABLE I

Simulation parameters

\begin{tabular}{ll}
\hline Bar length & $631.9 \mathrm{~mm}$ \\
Bar radius & $5 \mathrm{~mm}$ \\
Bar material density & $2.74 \mathrm{e}-6 \mathrm{~kg} / \mathrm{mm} 3$ \\
Young's Modulus & $7.1705 \mathrm{e} 4 \mathrm{~N} / \mathrm{mm} 2$ \\
Poisson's Ratio & 0.33 \\
Horizontal strings length & $346.4 \mathrm{~mm}$ \\
Vertical strings length & $510.6 \mathrm{~mm}$ \\
Spring stiffness & $15 \mathrm{~N} / \mathrm{mm}$ \\
Damping coefficient & $10 \mathrm{~N} . \mathrm{s} / \mathrm{mm}$ \\
Preload in strings & $50 \mathrm{~N}$ \\
External load & $100 \mathrm{~N}$ \\
\hline
\end{tabular}

As shown in Fig. 4, as the compressive force was applied on the structure, the top of structure was rotated anticlockwise. Applied load caused the motion in bar and they are displaced from their original location and also the original height of the structure is decreased under the effect of compressive loads. 


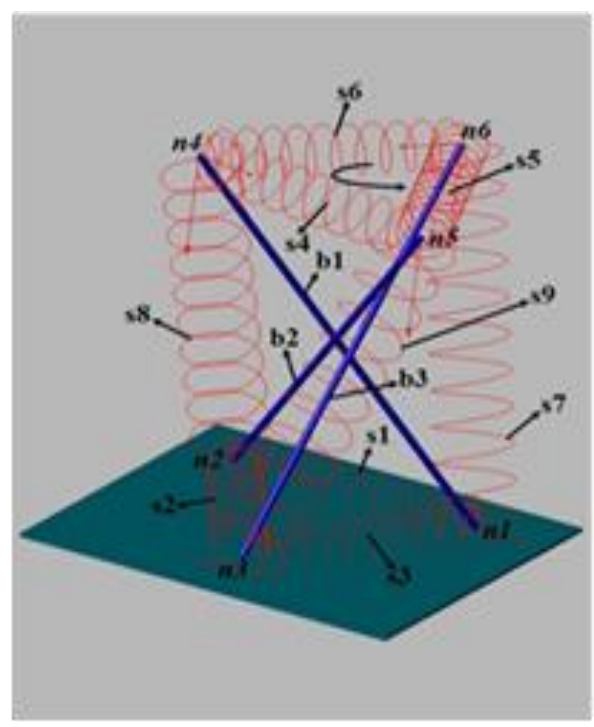

Fig. 4. Three bar tensegrity structure after applying compressive load

For analyzing the displacement of bars, we will see the displacement of center of mass of each bar because for a robotic structure, movement of center of mass cause the motion in overall structure until it reaches to another stable position. Therefore, movement of center of gravity of bar 1 , bar 2 and bar 3 in $\mathrm{X}, \mathrm{Y}$ and $\mathrm{Z}$ directions are shown in Fig. 5, 6 and 7 respectively.

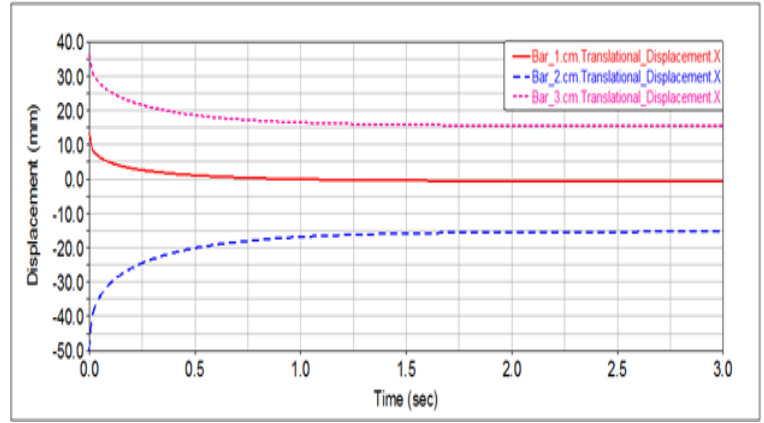

Fig. 5. Displacement of center of gravity in $\mathrm{x}$ direction of each bar

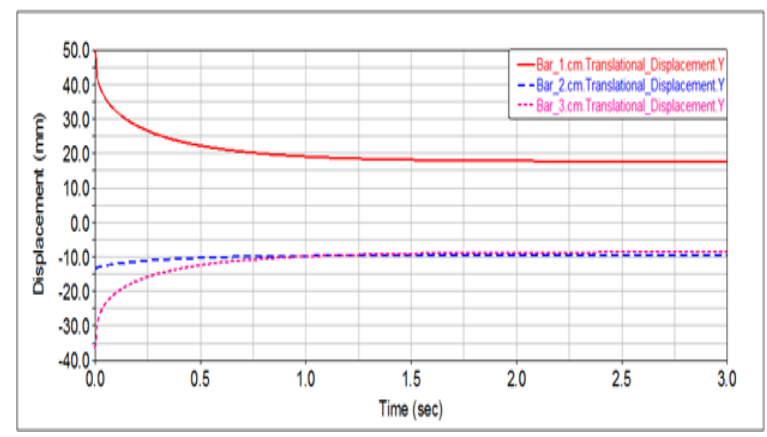

Fig. 6. Displacement of center of gravity in y direction of each bar

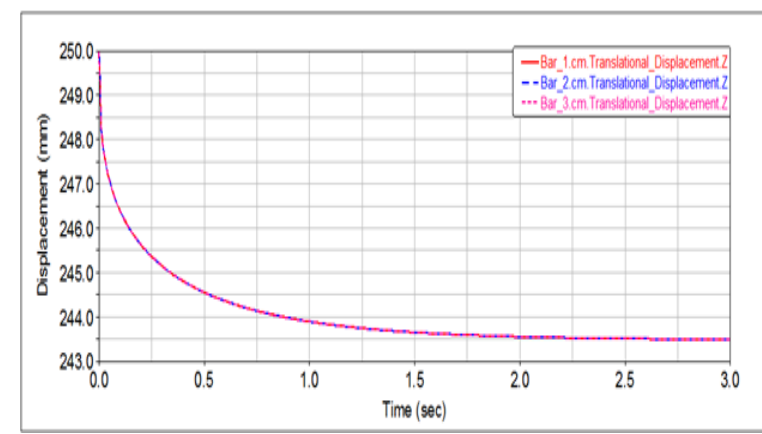

Fig. 7. Displacement of center of gravity in $\mathrm{z}$ direction of each bar

For a robot, it is necessary that the bars should not come in contact with each which will cause hindrance while locomotion therefore a certain distance is necessary for the overall robot motion. The distance between each bar center after applying the load is shown in Fig. 8 .

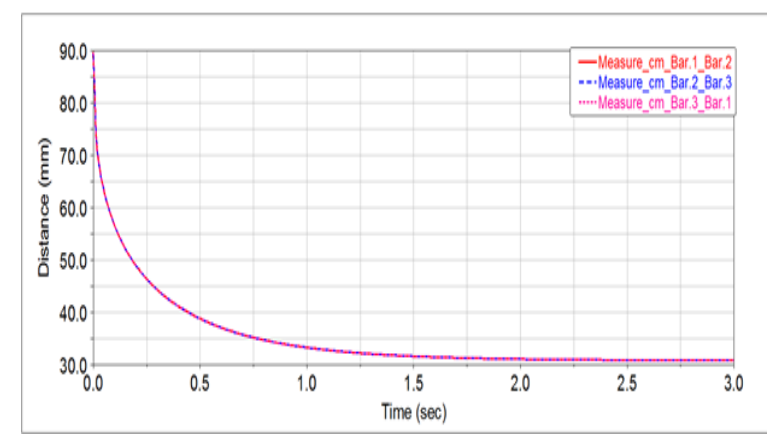

Fig. 8. Distance between center of mass of each bar

The external force also caused the deformation in all the strings of the structure. For strings 1,2 and 3 there was observed a negligible deformation as shown in Fig. 9. The deformation was same in strings 4, 5 and 6 as shown in Fig.10 while group of strings 7,8 and 9 had similar response among them as shown in Fig. 11.

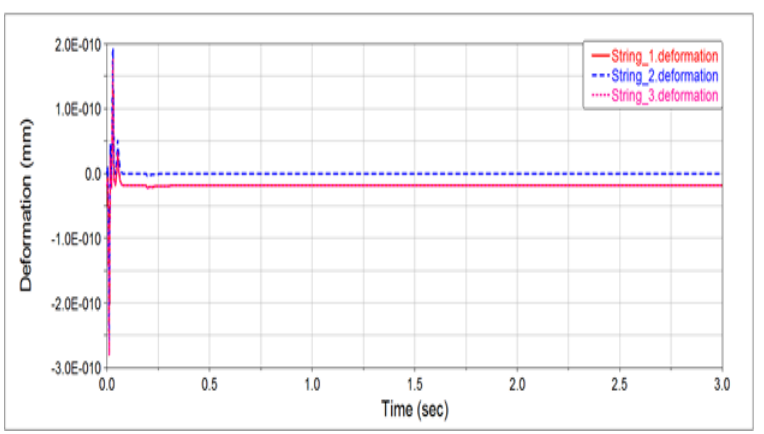

Fig. 9. Deformation in Strings 1, 2 and 3 


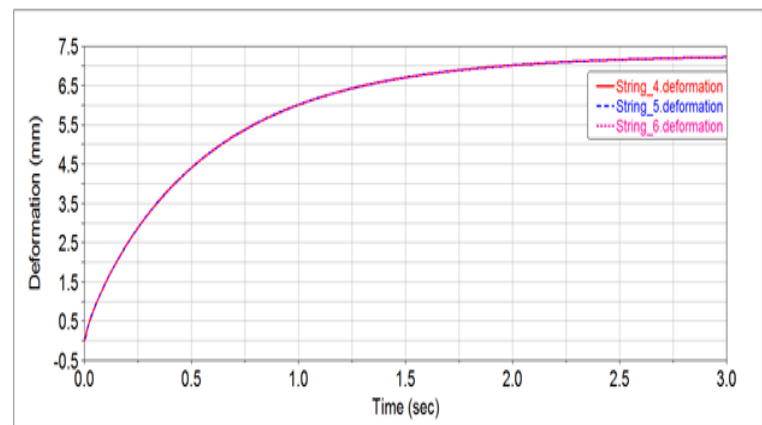

Fig. 10. Deformation in Strings 4, 5 and 6

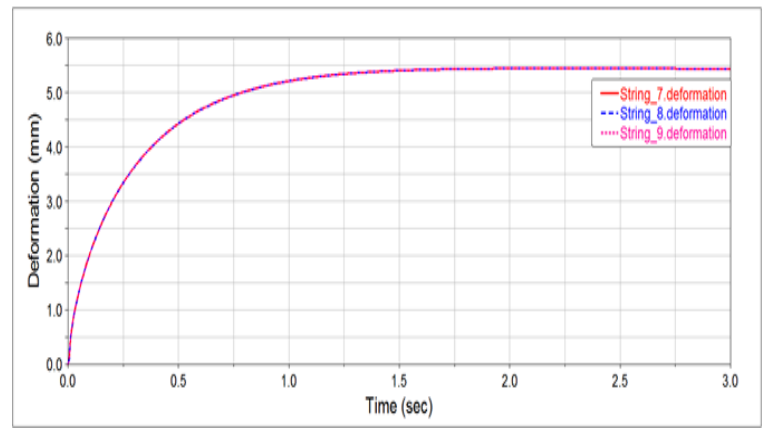

Fig. 11. Deformation in Strings 7, 8 and 9

Further in the next section the obtained results are tabulated and discussion is carried out.

\section{RESUlTS AND DisCUSSION}

The bars are the main component of the tensegrity structure because they possesses the primary mass and in a robotic application their movement will result in the overall locomotion of the robot. Disturbance in center of mass results in motion of a robot, therefore we observe from the graphs in Fig. 5, 6 and 7, the displacement of center of gravity of each bar of three bar tensegrity which are listed in the table below:

TABLE 2. BARS CENTER OF MASS DISPLACEMENT

\begin{tabular}{cccc}
\hline & $\begin{array}{c}\text { Displaceme } \\
\text { nt in X (mm) }\end{array}$ & $\begin{array}{c}\text { Displaceme } \\
\text { nt in Y (mm) }\end{array}$ & $\begin{array}{c}\text { Displaceme } \\
\text { nt in Z (mm) }\end{array}$ \\
\hline $\begin{array}{c}\text { Ba } \\
\text { r 1 }\end{array}$ & 13.92 & 31.17 & 5.94 \\
$\quad \mathrm{Ba}$ & 34.87 & 3.86 & 5.94 \\
$\mathrm{r} \mathrm{2}$ & & & \\
$\quad \mathrm{Ba}$ & 20.90 & 33 & 5.94 \\
$\mathrm{r} \mathrm{3}$ & & & \\
\hline
\end{tabular}

As shown in Table II, the overall structure displaced equally in $\mathrm{Z}$ direction while there are different displacements in $\mathrm{X}$ and $\mathrm{Y}$ directions. It is also be noted that structure is transformed into another stable position after displacement. These displacements can be controlled using linear motors in bars to give specific motion to robotic structure while maintaining equilibrium in a new position. Also, the bars should not come in contact with each other during displacement hence a safe distance of $30.90 \mathrm{~mm}$ is observed between mass centers of each bar as shown in Fig. 8.

With regard to the strings, a similarity of deformation was observed among the strings therefore they can be grouped as per similar deformation. For analyzing of the strings' deformations, we make strings groups as Group 1 which consists of strings s1, s2 and s3, Group 2 which consist of strings s $3, \mathrm{~s} 4$ and $\mathrm{s} 5$ and Group 3 which consist of strings s7, s8 and s9. The maximum deformations in each group of strings are listed in the table below:

TABLE 3. STRING DEFORMATIONS

\begin{tabular}{cc}
\hline Strings group & $\begin{array}{c}\text { Maximum deformation } \\
(\mathrm{mm})\end{array}$ \\
\hline Group-1 & $1.75 \mathrm{e}-10$ \\
Group-2 & 7.23 \\
Group-3 & 5.45 \\
\hline
\end{tabular}

As shown in Table III Group -2 strings experienced maximum deformation among all strings, which results in the anti-clockwise rotation of the structure as shown in Fig. 4. This rotational property can be utilized for forward locomotion of the robot and strings length can be controlled using a motor and pulley at the nodes end of the structure for the requisite movement of overall robot.

Thus, the simulation results illustrate that it is possible to construct a robotic structure based on basic three bar tensegrity structure by analyzing its dynamics and controlling the length of strings and the motion of bars. The resulting structure will be lightweight and can produce locomotion. Moreover, actuation in any member causes motion in whole structure therefore various combinations of members' actuation can be used to produce gait.

\section{CONCLUSION}

In this paper, a simple dynamic analysis of three bar tensegrity structure was carried out and its implementation in the field of robotics was discussed. Mathematical model was built for three bar tensegrity structure and node matrix, bar connectivity and string connectivity were defined. Using ADAMS simulation, motion of all bars and deformation in all strings were found. Finally, the results were discussed and it is concluded that a lightweight robotic structure based on tensegrity is possible. Similar methodology can be used for dynamic analysis of other type of complex tensegrity structures. As a future work, the physical robot can be built using three bar tensegrity structure to prove the dynamic analysis results achieved in this paper.

\section{ACKNOWLEDGEMENT}

This work is supported by the National Natural Science Foundation of China (Grant Nos. 51605111, 51675114 and 51875111). The authors also greatly acknowledge the constant support and motivation of Dr. Abdul Qadeer throughout this work. 


\section{REFERENCES}

[1] F. Buckminister, "Tensile integrity structures," United States, 1959.

[2] S. D. Guest, "The stiffness of tensegrity structures," IMA Journal of Applied Mathematics, vol. 76, no. 1, pp. 57-66, 2011.

[3] G. Castro and M. P. Levy, "Analysis of the Georgia Dome cable roof," in Computing in Civil Engineering and Geographic Information Systems Symposium, 1992, pp. 566-573: ASCE.

[4] M. P. Levy, "The Georgia Dome and beyond: achieving lightweight-longspan structures," in Spatial, Lattice and Tension Structures, 1994, pp. 560-562: ASCE.

[5] V. SunSpiral et al., "Tensegrity based probes for planetary exploration: Entry, descent and landing (EDL) and surface mobility analysis," International Journal of Planetary Probes, vol. 7, p. 13, 2013.

[6] F. Hiroshi, "Concept of deployable tensegrity structures in space application," International Journal of Space Structures, vol. 7, no. 2, pp. 143151, 1992.

[7] D. E. Ingber, "The architecture of life," Scientific American, vol. 278, no. 1, pp. 48-57, 1998.

[8] J. Zhang, S. Guest, and M. Ohsaki, "Symmetric prismatic tensegrity structures: Part I. Configuration and stability," International Journal of Solids and Structures, vol. 46, no. 1, pp. 1-14, 2009.

[9] R. Connelly, "Tensegrity structures: why are they stable?," in Rigidity theory and applications: Springer, 2002, pp. 47-54.

[10] R. E. Skelton, "Designing minimal-mass tensegrity telescopes of optimal complexity," Astronomy, SPIE Newsroom, 2013.

[11] M. B. Chandio, A. Luo, Y. Li, S. Khushak, A. Raza, and "Dynamic Similarity of Six Bar Ball Tensegrity Structure in Compression and Expansion Processes," Journal of Harbin Institute of Technology, 2019.

[12] A. Luo, S. Che, and H. Liu, "The driving of the sixbar tensegrity robot," in 2017 IEEE International Conference on Robotics and Biomimetics (ROBIO), 2017, pp. 640-645: IEEE.

[13] C. Paul, J. W. Roberts, H. Lipson, and F. V. Cuevas, "Gait production in a tensegrity based robot," in ICAR'05. Proceedings., 12th International Conference on Advanced Robotics, 2005., 2005, pp. 216-222: IEEE.

[14] R. E. Skelton and M. C. de Oliveira, Tensegrity systems. Springer, 2009.

[15] R. Motro, Tensegrity: structural systems for the future. Elsevier, 2003.

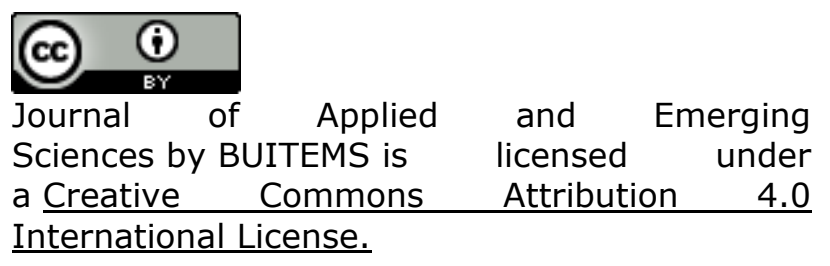

